\title{
Des écoles de pêche aux lycées maritimes et aquacoles
}

Denis Biget

\section{(2) OpenEdition}

1 Journals

Édition électronique

URL : https://journals.openedition.org/tc/1351

DOI : $10.4000 /$ tc. 1351

ISSN : 1952-420X

Éditeur

Éditions de l'EHESS

\section{Édition imprimée}

Date de publication : 1 juin 2005

ISSN : 0248-6016

\section{Référence électronique}

Denis Biget, «Des écoles de pêche aux lycées maritimes et aquacoles », Techniques \& Culture [En ligne], 45 | 2005, mis en ligne le 22 mai 2008, consulté le 29 septembre 2022. URL : http:// journals.openedition.org/tc/1351; DOI : https://doi.org/10.4000/tc.1351

Ce document a été généré automatiquement le 29 septembre 2022.

Tous droits réservés 


\title{
Des écoles de pêche aux lycées maritimes et aquacoles
}

\author{
Denis Biget
}

\section{NOTE DE L'ÉDITEUR}

Dans un premier article, Denis Biget retrace l'histoire des premières écoles de pêche maritime nées dans les années 1895 pour rationaliser et moderniser les activités halieutiques mais aussi pour encadrer les populations littorales. Cependant, et malgré cette volonté de rationalisation, les marins pêcheurs d'aujourd'hui persistent à apprendre leur métier sur le tas, parallèlement aux enseignements scolaires devenus obligatoires pour exercer leur métier. Dans un deuxième article tiré d'une enquête de terrain, le même auteur nous montre que c'est encore le point de vue d'une majorité des élèves des modernes Lycées maritimes et aquacoles, qu'ils soient ou non issus du milieu maritime.

1 L'idée de cette étude trouve son origine dans mon travail de maîtrise de sociologie (Biget 1992) qui portait sur l'origine du choix du métier de marin pêcheur. J'avais proposé l'hypothèse d'une affiliation culturelle des jeunes au milieu de la pêche telle que leur univers de pensée et de vie favorisait leur entrée dans ce secteur, grâce à un ensemble de représentations sociales sur le métier, le rapport au monde et le processus d'apprentissage.

2 Constatant dans les discours des jeunes en formation pêche en 1992, que le métier s'apprend encore sur le tas, par socialisation, mais que l'école et le diplôme sont indispensables, je me suis demandé comment et pourquoi la transmission d'un savoir a progressivement laissé la place à la transmission scolaire et institutionnalisée des connaissances.

3 Le métier reste essentiellement un ensemble de savoirs et de techniques qui ne s'acquièrent qu'au cours de l'action et au fil du temps, sans pouvoir être rassemblés dans un corpus de savoirs et de connaissances précises ; c'est plutôt, pour emprunter 
une expression de la sociologue Geneviève Delbos (Delbos \& Jorion 1984), un «savoir y faire " adaptable aux situations variées et imprévisibles qu'impose la nature aux producteurs -absence de la ressource, caprices du temps, des marchés, des hommes, des techniques. Il nous faut donc chercher comment le savoir dispensé dans ces écoles est construit par les responsables de la formation.

4 L'école de pêche n'échappe pas à la règle avancée par Emile Durkheim (1992: 46) qui énonce que les systèmes d'éducation dépendent «de la religion, de l'organisation politique, du degré de développement des sciences, de l'état de l'industrie». Concernant un petit nombre d'élèves, elle montre néanmoins comment une société a créé, pour un secteur particulier de sa population, une partie spéciale de son système éducatif en vertu de principes généraux et communs qui animent la société dans son ensemble.

5 En dehors de son rôle pédagogique, il faut comprendre sur quels principes le système de formation professionnel des pêches maritimes s'est formé, et quels sont les liens avec l'ensemble des courants de pensée de la société de l'époque. On s'aperçoit alors que le programme des connaissances requises pour exercer le métier et accéder aux prérogatives qu'il confère ne découle pas d'un savoir sui generis mais répond aux nécessités politiques, économiques et idéologiques des groupes concernés aux différentes époques de l'histoire.

6 Aucune source disponible ne traite précisément de ce système de formation. Cependant, nombreux sont les ouvrages ou articles sur l'histoire de la pêche qui le mentionnent, plus ou moins brièvement. Pour les raisons exposées plus haut, le système de formation est au cœur de l'évolution des activités halieutiques.

7 L'école est le lieu où se sont cristallisés les différents courants, les incertitudes, les professions de foi et aussi les réalisations les plus concrètes en recouvrant de la caution institutionnelle et scientifique le paradoxe de l'activité de pêche, à la fois assise dans la tradition et parfois à la pointe de la modernité technique, économique et sociale. La pêche a été adaptée aux nouvelles exigences économiques tout en conservant un certain nombre de pratiques (gestes, rémunération à la part, marché...). Ce n'est pas par hasard que la formation dans ce secteur prend naissance à la fin du XIXe siècle au moment où la pêche entre dans un système de production et de commercialisation industriel.

8 En gardant présente à l'esprit l'apparente contradiction entre savoirs pratiques et connaissance scolaire, j'ai essayé de comprendre comment ont été créées ces écoles, comment ont été élaborés leurs programmes et quels étaient les objectifs de leurs créateurs, leur formation idéologique ou politique. Comme si le raisonnement avait adhéré à la réalité -ce qui serait une réussite épistémologique inespérée-, je n'ai pu faire l'histoire des écoles de pêche, de leurs promoteurs, de leurs programmes, de leur fonctionnement et de leur équipement sans revenir à ceux à qui elles s'adressaient.

L'enseignement hydrographique

9 Les limites d'une telle étude ne nous permettent pas de faire des recherches approfondies dans les documents d'archives concernant la formation des gens de mer. Toute la partie couvrant la période du XVI ${ }^{\mathrm{e}}$ siècle au XvIII ${ }^{\mathrm{e}}$ siècle est extraite de l'ouvrage d'Alain Cabantous (1991: 233-236 ; 461-466). La partie relative à la période la plus récente, le XIX repose sur l'analyse d'archives du Finistère et du Morbihan. La période concernant la création des écoles d'apprentissage maritime et leur évolution, s'appuie 
sur l'analyse de coupures de presse et documents divers que m'a confiés Geneviève Delbos (CNRS), et sur ses précieuses remarques sur ce sujet.

On ne trouve pas trace d'un enseignement professionnel des pêches maritimes avant la fin du xIX siècle. On trouve néanmoins des cours destinés aux ostréiculteurs dès 1862 à Concarneau et à Auray. Jusque-là, un compagnon pêcheur ou matelot pouvait se présenter devant un jury compétent afin d'être reconnu pilote, maître de pêche ou patron au cabotage.

L'exigence de la preuve de connaissance nécessaire à l'exercice du métier de marin peut être datée de 1584 : l'article 86 d'une Ordonnance de Charles IX prescrit que nul ne peut être embarqué sur un navire de commerce en qualité de maitre, patron ou pilote sans avoir été interrogé sur le fait de navigation par deux anciens maîtres en présence des Officiers de l'Amirauté. Au grand cabotage, il a suffi «que deux anciens capitaines de port aient dit qu'il était capable de naviguer et de conduire un bateau " pour que Jean Delabbyt fût reçu capitaine en 1695 (Cabantous 1991 : 234). D'autres devront prouver qu'ils sont en possession de cartes, cours, moyens de calcul des marées et autres outils pour la navigation.

Déjà, face à ce monopole de l'empirisme, Digard de Kerguette, à la fin du XVII siècle, s'insurgeait avec une certaine exagération contre l'insuffisance de connaissances théoriques des futurs maitres de navire. Depuis longtemps en effet, autorités urbaines et personnes privées des villes portuaires s'efforçaient d'assurer un enseignement hydrographique.

L'Ordonnance de 1629, connue sous le nom de Code Michaud, reprenant les prescriptions de celle de 1584, ajoute aux deux examinateurs prévus le professeur d'hydrographie dont la fonction vient d'être créée. Dans ce texte, le roi s'engageait à procurer trois fois par semaine des leçons publiques et gratuites aux marins. L'enseignement maritime prend naissance et les écoles officielles remplacent progressivement les écoles privées dont la plus connue était celle de l'abbé Descelliers à Dieppe, qui dès 1661 devient École royale. La Constitution de 1791, qui proclame l'instruction publique, prive les écoles chrétiennes des ressources financières et compromet la formation professionnelle en même temps qu'elle supprime les corporations ; un an plus tard, les congrégations religieuses sont interdites, mais elles peuvent néanmoins ouvrir des écoles privées. Les toutes premières entreprises dont nous avons parlé ont rencontré un plus ou moins grand succès, notamment à cause de l'insuffisante compétence des professeurs et de l'irrégularité de la scolarité qui alternait avec des périodes d'embarquement (en 1724, 53,7 \% des élèves de l'école du Havre sont inscrits depuis plus de quatre ans) (Cabantous 1991: 235). La forte reproduction du groupe de capitaines privilégiant la transmission familiale des connaissances n'empêche pas une sensible augmentation du nombre des brevetés (+13\% de 1691 à 1791) (Cabantous 1991: 236). La fréquentation des écoles d'hydrographie augmente sous l'influence des impératifs commerciaux, de l'amélioration des techniques et de la volonté de l'administration (Ordonnance du 16 Janvier 1786).

Le XVIII ${ }^{\mathrm{e}}$ siècle est l'époque d'une certaine démocratisation de l'instruction. Face à une intellectualisation croissante des professions, les corporations et l'apprentissage sur le tas cèdent le pas à l'ouverture de cours professionnels, dont les cours de navigation donnés par les écoles chrétiennes qui, sous l'influence du mouvement piétiste, tendent à ouvrir l'enseignement sur la vie, en accueillant les enfants des couches les plus 
défavorisées. En 1883, après une longue succession de décrets et ordonnances, une réforme créera les "Élèves de la Marine Marchande ", institution qui a pour objet de faciliter les carrières des jeunes d'origine terrienne, sans obligation préalable de navigation. Au début du xxe siècle, on trouve les écoles de navigation maritime et les écoles professionnelles et techniques maritimes secondaires (réorganisées en 1919) sur tout le littoral. En deux ans, elles préparent les jeunes inscrits maritimes, âgés de treize ans au moins, aux carrières de capitaine au long cours, maître de cabotage ou patron au bornage. Aujourd'hui, quatre écoles nationales de la Marine marchande (ENMM) sous tutelle des structures ministérielles chargées de la mer et des Transports se partagent la formation des marins de commerce.

Instruire et former les pêcheurs

15 Si l'augmentation de la scolarisation a favorisé une mobilité ascendante -pas toujours de façon égalitaire- dans le groupe des capitaines, il n'en a pas été de même chez les pêcheurs dont l'analphabétisme, associé au poids des habitudes familiales et des parlers locaux, fut un obstacle à la scolarisation et à l'ascension sociale. La volonté de christianiser les gens de mer par la voie de l'instruction, aux XVII ${ }^{\mathrm{e}}$ et $\mathrm{XVIII}{ }^{\mathrm{e}}$ siècles, va entraîner l'ouverture d'écoles gratuites pour les enfants de marins et de pêcheurs.

Les écoles de l'hôpital du Havre (16 mai 1669) ou de Dunkerque (1737) avaient pour objectif de renforcer l'ordre maritime et la stabilité sociale par l'éducation chrétienne et l'enseignement à l'obéissance. Nombreuses furent les villes qui firent construire des écoles pour instruire et éduquer les matelots. Les directives ministérielles de de Castries officialisaient l'acquisition de connaissances théoriques pour le brevet de maître de pêche, répondant en cela à la volonté des maîtres de pêche les plus aisés de donner à leurs enfants et aux aspirants à la maîtrise une instruction élémentaire. Ces tentatives de scolarisation religieuse au départ, professionnelle par la suite, ont davantage porté leurs fruits chez les capitaines, culturellement favorisés.

Chez les pêcheurs, le résultat est moins remarquable. L'alphabétisation -révélée par l'indicateur de "sachant-signer »- y est encore faible. Les communautés de pêcheurs où dominait la culture orale, où les jeunes embarquaient très tôt ou travaillaient avec leur mère à la pêche à pied sur les grèves, constituant ainsi un apport économique indispensable, connaissaient un mode d'acculturation qui n'avait pas besoin de l'écrit. Les pêcheurs, contraints par leur situation économique à négliger l'instruction de leurs enfants, étaient donc en position défavorable par rapport à l'enseignement général. En outre, les modes de socialisation communautaires qu'imposaient les nécessités économiques et dont l'un des principes était la transmission familiale, dispensaient ces groupes de marins d'avoir recours à l'organisation scolaire et théorique pour l'acquisition des connaissances nécessaires à l'exercice de leur métier.

18 Cette époque héritière des Lumières attache une grande importance à l'enseignement professionnel afin de permettre un développement de l'industrie sur les voies de la science et de la raison. Elle veut faire entrer la connaissance dans les campagnes en reliant l'instruction et le métier par la formation " par accoutumance au travail ».

Le début $\mathrm{du} \mathrm{xIX}^{\mathrm{e}}$ siècle voit naître l'instruction populaire et les premières sociétés d'encouragement pour l'industrie et l'instruction. Sous la Restauration et la Monarchie de Juillet, la bourgeoisie industrielle cherche à accroître les compétences techniques des ouvriers et à leur inspirer le respect de l'ordre social. Les congréganistes ont retrouvé tout pouvoir et, dans le cadre de la loi Guizot, sont créées des écoles de mousses (comme à Bordeaux en 1839) pour « convertir en marins exercés une foule 
d'enfants livrés à tous les désordres du vagabondage ». C'est aussi la période du développement des fermes-écoles et des premiers enseignements agricoles.

Sous le Second Empire, l'apprentissage est en crise. Les Expositions de Londres et de Paris l'avaient déjà signalé. La défaite de 1870 montre la supériorité industrielle de l'Allemagne et remet en cause le libéralisme économique. La société s'engage dans un mouvement d'intégration qui marque l'école au travers des grandes lois de Ferry, et surtout, pour ce qui nous concerne, des lois sur l'enseignement professionnel et technique, en particulier la création des écoles d'apprentissage manuel (11 décembre 1880). La IIIème République prendra des mesures (travaux manuels ${ }^{1}$, organisation de la formation professionnelle...) pour y remédier et ouvrira les écoles d'apprentissage afin « d'acheminer les élèves vers les métiers auxquels les prédestine le milieu naturel ».

L'enseignement des pêches au XIX ${ }^{\mathrm{e}}$ siècle

21 La situation des marins pêcheurs par rapport à l'instruction reste sensiblement la même au cours de cette période. En 1865, les autorités maritimes de Lannion déplorent encore l'analphabétisme des pêcheurs. En 1896, elles prennent des mesures pour l'obtention du Certificat d'études des Inscrits maritimes. L'arrêté du 20 septembre 1898 fixe la liste des écoles « où devront être données des leçons de choses appropriées à la profession de pêcheurs » (Roignant 1992 : 62-63). La Société d'enseignement technique et professionnel des pêches maritimes (créée en 1895) adresse, en 1904, à l'Inspection primaire de Lannion, les ouvrages et matériels à distribuer aux écoles du littoral. Le succès reste très relatif puisque, dans le Bulletin des pêches maritimes de 1912, on lit :

«La pêche est un métier qu'il faut aborder très jeune pour y prendre goût. Les gamins de neuf à dix ans commençaient jadis leur apprentissage de bonne heure et restaient à la mer; abandonner le bateau aurait été une véritable désertion. Actuellement, lorsque les enfants ont leur Certificat d'études, les parents estiment qu'ils sont trop instruits pour risquer si gros et gagner si peu. »

Pour la grande pêche à la morue en Islande, par exemple, ce n'est que le 20 janvier 1914 qu'est institué le brevet de patron de pêche avec obligation d'études avant examen.

Au sortir de la crise qui sévit de 1873 à 1895, le gouvernement cherche dans la science le moyen de relancer l'économie. Les congrès réunissent savants, hommes politiques, industriels et philanthropes. Le Congrès national des pêches côtières préconise la création d'écoles de pêche. L'arrêté du 20 septembre 1893 impose l'enseignement de la lecture des cartes marines dans les écoles primaires du littoral.

C'est vers 1895 que, à la suite d'un congrès de la Société de Sauvetage tenu à Saint-Malo, l'idée de la formation professionnelle des pêches maritimes se précise. Le Comité d'exécution de cette société décide de créer des écoles de pêches, délaissant momentanément la question du sauvetage. Pour cela, elle fonde, les 11 et 27 février 1895, la Société d'Enseignement Professionnel et Technique des Pêches Maritimes (SEPTPM) dont les statuts sont approuvés par le ministère de l'Intérieur le 16 mai 1895 (Biget 1997). L'objet de cette société est de «donner aux pêcheurs des connaissances suffisantes qui leur permettront d'exercer leur périlleux métier avec moins de dangers, et d'augmenter leurs bénéfices de façon à pouvoir s'assurer contre la maladie, les accidents et la vieillesse ».

Le président de la SEPTPM, Emile Cacheux, fait partie de ces hommes du XIX architectes, hygiénistes, moralistes, qui cherchent à améliorer les conditions de vie "dégradées », pensent-ils, par l'urbanisation et l'industrialisation qui engendreraient insalubrité, promiscuité et dépravation morale. Toute leur action repose sur 
l'opposition entre les valeurs économiques et morales de la ville et de la campagne. Également Président de la Société de sauvetage, Emile Cacheux est le fondateur des cités ouvrières des Lilas, du boulevard Kellerman, de l'impasse Boileau, médaille d'or de l'Exposition d'Hygiène de Londres et de l'Exposition universelle de Paris en 1889. Il est l'auteur de plusieurs ouvrages: Les habitations ouvrières à la fin du 19ème, L'économie pratique, (crèches, asiles, écoles maternelles, cercles, bains, lavoirs), le Manuel de sauvetage, et il signe Habitations ouvrières en tous pays, avec Emile Muller, professeur à l'École des Arts et Manufactures, ancien président de la Société des Ingénieurs civils, architecte des cités ouvrières de Mulhouse.

La liste des personnalités membres de la SEPTPM se compose d'hommes politiques, de juristes, d'éminents professeurs et docteurs de l'Université, de hauts gradés de l'armée et de la marine, de savants français et étrangers, d'inspecteurs des pêches, des premiers "aquiculteurs". On trouve aussi les noms de Ferdinand Buisson, directeur de l'enseignement primaire, de Pierre Loti, du Baron Arthur de Rothschild, de Théophile Villard, président d'honneur de la Société centrale du travail professionnel. Tous ces hommes, sans oublier Madame la «Doctoresse » Pokitonoff, de l'Université de SaintPétersbourg, sont, quelles que soient leurs appartenances idéologiques, animés par le même souci de favoriser l'intégration des classes populaires dans la société (Raymond 1968).

Le monde de la pêche n'échappe pas à ces observateurs-réformateurs. Depuis 1850-1860 (Vauclare 1988; Mollat 1988) environ, on assiste à une croissance des activités de pêche, qui entrent dans l'ère de l'industrialisation grâce aux innovations techniques, à bord (chaluts à panneaux, treuil à vapeur, motorisation) comme à terre (conserve, glace, chemin de fer), et qui demandent de gros investissements et engendrent une concentration des capitaux. Cette évolution s'accompagne d'une forte urbanisation, mais aussi d'une prolétarisation des populations littorales. Invoquant le besoin de faciliter l'entrée de la pêche dans l'économie moderne tout en évitant ses effets pervers, hommes politiques et hommes de science s'accordent pour reconnaître l'utilité d'une formation des pêcheurs dont l'empirisme et l'individualisme constituent selon eux un obstacle au progrès. C'est aussi un moyen d'éviter que les pêcheurs n'adhèrent trop facilement aux idées collectivistes qui tentent difficilement de pénétrer ce monde que l'industrialisation rapproche de plus en plus de la société ouvrière socialisante, tout au moins dans les grands ports.

28 Le but de la Société d'enseignement professionnel et technique des pêches maritimes est double: donner aux marins des connaissances en navigation, pour éviter les naufrages et accidents, leur permettre d'accroître la rentabilité, pour contribuer à développer l'exploitation des océans, source de richesses qu'il n'est pas besoin d'ensemencer, et qui peut permettre de remédier aux situations de crise, pour le bien de l'humanité. Le but est aussi de consolider la place de l'industrie des pêches françaises face à la concurrence étrangère. Organiser la profession en développant le mouvement coopératif pour aménager des paliers de financement et de commercialisation, et donc de faire participer les pêcheurs les plus passéistes au progrès, tout en améliorant leurs conditions de vie et de travail. Nous sommes en pleine époque positiviste et de croyance au progrès.

À ces deux grands principes s'ajoutent deux missions : occuper les jeunes gens en les défendant contre l'oisiveté et la débauche auxquelles le temps à terre les expose, mais 
aussi, et cela fait partie du programme, préparer les jeunes aux équipages de la flotte, en leurs facilitant l'accès aux écoles des spécialités de la marine de guerre².

La première école de pêche est fondée à Groix ${ }^{3}$ (avant même l'autorisation ministérielle), grâce à une subvention de la SEPTPM, du conseil municipal de Groix, sous le patronage de la Chambre de Commerce du Morbihan et avec l'aide financière des ministères du Commerce et de la Marine. La direction de l'école est confiée à Victor Guillard (1896), professeur d'hydrographie à Lorient, chevalier du Mérite agricole, exmembre du conseil départemental de l'Instruction Publique. Il est connu depuis 1887 pour ses expériences sur les techniques de navigation et de pêche. Au départ, les cours sont dispensés sous forme de conférences, sur des questions de navigation essentiellement, mais aussi sur les gréements, la conservation du poisson, la réglementation des pêches, l'hygiène (et les dangers de l'alcool), la natation, l'économie sociale pour propager les institutions de prévoyance, et comprennent un enseignement de français et d'arithmétique. Ces conférences ont lieu toute l'année, sauf en été et automne, saison de pleine activité; elles sont ouvertes aux Inscrits maritimes d'au moins treize ans, matelots et patrons. L'enseignement est gratuit, les cours ont lieu le matin et le soir.

La première conférence est prononcée le 16 mai 1895 par Victor Guillard, en présence du maire et du commissaire de l'Inscription maritime, devant 23 élèves dont 14 patrons. Le directeur de l'école publique et ses trois adjoints y assistent et apportent leur concours. Devant l'assiduité et le succès des élèves les plus jeunes (car les anciens sont peu intéressés, voire hostiles), M. Guillard comprend avec satisfaction que cette entreprise est appelée à se développer. L'école reçoit rapidement chaises, tables, cartes, compas et sextants.

Voici la description qu'en donne Anatole Le Braz dans son ouvrage Navigation morbihannaise chez les groisillons, en 1900 :

«Une classe quelconque : des bancs grossiers, des tachées d'encre et de goudron; ça et là des cartes, des instruments de marine suspendus aux parois. Au fond de la salle, une inscription, une seule, tracée en lettres noires sur la chaux de la muraille "l'alcoolisme, voilà l'ennemi"."

Ayant assisté à quelques cours, il s'étonne devant ces rustres pêcheurs qui essaient de graver la leçon dans leurs « crânes de primitifs » [sic]. Dans son rapport au commissaire général de l'Inscription Maritime, Victor Guillard conclut :

«De précieuses existences nécessaires à la défense du pays seront ainsi conservées; l'industrie si nationale et si patriotique de la pêche se fera avec plus d'intelligence, et prendra un nouvel essor; les parcours en mer seront moindres [...]; et viennent les jours de la suprême lutte, la marine de guerre trouvera parmi la population instruite de Groix, si habile déjà dans la connaissance des sondes, toute une pépinière d'intrépides et intelligents pilotes. » (Guillard 1896)

Mais les pêcheurs n'apprécient pas tous qu'on les prenne pour des ignorants. Par la voix du journal catholique la Croix de Groix, ils écrivent :

«Les patrons actuels sont des ignorants en fait de navigation : il faut les envoyer à l'école. À l'école de qui ? De M. Guillard. Un patron n'est capable de diriger son bateau que s'il possède un brevet. Qui lui donnera son brevet? Un maître d'école. » (Duviard 1992)

L'école de M. Guillard est laïque, est-il besoin de le préciser ? J’y reviendrai.

La réussite de cette première conférence engage la SEPTPM à créer une seconde école aux Sables-d'Olonne, lors de la séance du conseil municipal du 26 mai 1895, sous le nom 
d'École Municipale d'Enseignement Technique et Professionnel des Pêches Maritimes des Sables-d'Olonne qui est la seule, avec celles de l'île d'Yeu et de Fécamp aujourd'hui encore, à exister sous une forme privée.

De 1895 à 1910, des écoles voient le jour à Boulogne, Dieppe, Fécamp, Le Havre, Honfleur, Villerville, Saint-Vaast-La-Hougue, St Quay-Portrieux, Camaret, Douarnenez, Tréboul, Audierne, Saint-Guénolé, Kerity-Penmarc'h, Lesconil, L'Ile Tudy, Concarneau, Le Croisic, La Rochelle, Fouras, Arcachon, Martigues, Marseille. Elles sont gérées selon des modalités diverses (Chambres consulaires, municipalités, sociétés...).

Leçons de choses

Les écoles de pêche ne se ressemblent pas. Dans certains cas, ce que l'on a appelé école de pêche consistait en cours professés par le directeur de l'école primaire en plus de son enseignement, assisté d'un ou de plusieurs instituteurs-adjoints, spécialement destinés à l'enseignement nautique lorsque le nombre de pêcheurs et l'importance de la pêche l'exigeaient. Enthousiasmé par les résultats de l'enseignement professionnel en France, l'Inspecteur général de l'Instruction publique, M. Coutant, obtient le vote de l'arrêté du 22 septembre 1898 qui institue les Leçons de choses appropriées au métier de marin dans les écoles communales du littoral. Ces cours étaient facultatifs ou obligatoires selon que l'école recevait beaucoup ou peu d'enfants appelés à devenir marins. Certaines écoles ont retrouvé les cours nautiques qu'elles avaient perdus un ou deux ans plus tôt. Il n'est pas aisé de savoir exactement quand ces cours ont cessé, puisque pour certaines écoles, on n'en trouve plus trace dans les archives après 1925, comme à Billiers (Morbihan), alors que cette école possède aujourd'hui encore un exemplaire de Ce qu'il faut savoir sur la mer, la navigation et la pêche maritime datant de 1950. (Une circulaire ministérielle de 1943 préconise la réouverture de deux heures hebdomadaires d'enseignement nautique à l'école primaire de Morlaix). Par contre, d'autres écoles perdent ces cours presque aussitôt, comme Penestin, le Tour du Parc, Noyalo, Le Hezo, zones d'agriculture et de marais salants dans le Morbihan, ou encore, dans le Finistère, Bénodet qui ne compte que quatre fils de marin, la côte nord de Goulven à Porspoder où l'on compte $87 \%$ de cultivateurs qui s'inscrivent à la pêche juste le temps de la récolte du goémon, contre seulement $7 \%$ d'Inscrits maritimes professionnels. On trouve un certain nombre de lettres d'instituteurs qui demandent à l'Inspecteur d'Académie de ne pas continuer ces cours, ou de les remplacer par des cours d'agriculture, en raison du nombre important d'enfants de cultivateurs.

Néanmoins, si les cours d'enseignement nautique donnent entière satisfaction au point que Camaret reçoit un deuxième instituteur adjoint et Audierne un septième, on commence à déplorer la trop grande dépendance de l'école de pêche par rapport à l'école publique. L'école de pêche n'a pas de réelle existence. La répartition des tâches entre l'instituteur chargé des cours de pêche, l'instituteur primaire et les intervenants extérieurs (officiers, pêcheurs...) ne va pas sans problème ; c'est le directeur de l'école publique qui gère les subventions affectées à l'école de pêche. Les querelles politiques et religieuses locales nuisent à l'unité de l'enseignement (et dégénèrent même en pugilat comme à Camaret en 1912 !) ${ }^{4}$.

L'idée d'une organisation de l'enseignement des pêches conduisant à son autonomie se précise. Les exemples de plusieurs pays d'Europe dotés d'écoles d'État, ou de l'Allemagne avec sa Deutch seefiserien verien qui possède vingt-neuf écoles sur les bords de la Baltique et dix-sept sur les bords de la mer du Nord, sont cités. L'État comprend trop l'importance de la pêche dans l'économie nationale pour laisser échapper toute 
cette partie de la population à tout contrôle. La trop grande diversité des modes de gestion des écoles de pêche est préjudiciable à leur fonctionnement (obtention de subventions, organisation des programmes...). Plusieurs projets de loi vont se succéder pour réorganiser l'enseignement des pêches vers plus de cohérence, en renforçant son lien de tutelle avec l'État par le biais de l'administration préfectorale. Le préfet est le maillon entre le Comité central permanent de l'enseignement professionnel des pêches, organe du ministère, et le Comité départemental, composé de fonctionnaires territoriaux, de responsables des chambres consulaires, d'industriels et de professionnels.

41 L'arrêté du 24 septembre 1909 signé par le sous-secrétaire d'État à la Marine, Cherron, s'applique à préciser le fonctionnement de la préparation en matière de pêche et de service de la flotte armée. Les organismes gestionnaires des écoles de pêches (sociétés nautiques de régate, de natation, d'anciens marins...) deviennent des Sociétés Agréées par la Marine (SAM). Elles doivent faire déclaration conformément à la loi sur les associations du 1er juillet 1901, ce qui les autorise à demander des subventions (et pour leurs membres, à recevoir des récompenses honorifiques...).

En 1913, malgré l'arrêté Cherron et les bons résultats des écoles, aucune suite n'est donnée à cette réorganisation. La loi Astier du 25 juillet 1919 sur l'organisation de l'enseignement technique et de l'apprentissage offre un cadre favorable à l'enseignement des pêches. Une circulaire ministérielle du 26 décembre 1924 adressée au préfet du Morbihan au sujet de l'école d'Etel précise que l'administration de la Marine laisse aux écoles de pêche et aux communes toute liberté pour organiser les cours selon les besoins de la population locale en vue de l'obtention du certificat de capacité et du brevet de patron récemment créés. Le ministre fait remarquer que l'arrêté Cherron mélange à tort préparation militaire et préparation à l'industrie de la pêche, au détriment de cette dernière. L'enseignement maritime se professionnalise et seuls les titulaires du Certificat d'Études Primaires peuvent se présenter au cours pour le brevet de patron (il invite par ailleurs à ne pas oublier les Leçons de choses de 1898). Dans le cadre de la loi Astier qui prévoit la création de commissions locales professionnelles chargées d'organiser les cours pour les besoins des industries locales, il faut ouvrir des écoles de pêche dans les ports les plus importants.

La pêche maritime est à cette époque dans une nouvelle phase de développement. La Première Guerre mondiale a favorisé la régénération des ressources, et les conditions sociales s'améliorent. Pourtant, dès 1928, les premiers signes de déclin apparaissent. À la raréfaction de la ressource s'ajoute le marasme économique général dû, en ce qui concerne la pêche, à une surproductivité et à un manque d'organisation des marchés. Autour de 1930, la pêche voit ses revenus et ses conditions de vie se dégrader et ses effectifs diminuer. Le ministre de l'Instruction Publique et des Beaux-Arts engage les recteurs et inspecteurs d'Académie à favoriser, consolider et développer la préparation à l'enseignement nautique dans les écoles primaires afin de ramener les populations littorales vers les métiers de la mer et de la pêche qui exigent désormais plus de connaissances théoriques et scientifiques que la seule expérience.

Du métier vers la profession

Le monde maritime, tellement diversifié selon les régions et les types d'activité halieutique, est le sujet de réflexions de deux courants idéologiques; chacun de ces courants tente d'investir le système éducatif des pêches maritimes. Pour reprendre la typologie de C. Vauclare (1988), il y a d'un côté le mot d'ordre des syndicats de gauche, 
qui préconise une organisation des pêches en termes de lutte des classes, et celui du catholicisme social et des syndicats chrétiens, avec le père Lebret, qui cherche à instaurer un cadre professionnel pour la défense du «bien commun ». Travaillant aussi bien auprès des populations (réunions, enquêtes, organisation de services, écoles) que des hautes instances de l'état (le père Lebret participe à la préparation de la loi sur le Comité central des pêches), ces deux courants conduisent à une transformation du métier en profession, sur la base du corporatisme en ce qui concerne le courant catholique. De la Première Guerre mondiale jusqu'aux années 1940, les écoles de pêche ont presque disparu. Quelques-unes végètent.

Encadrer les jeunes sous l'Occupation : les écoles d'apprentissage maritime

Sous l'Occupation allemande, le nord de la France a perdu pratiquement tout trafic maritime, la pêche est placée sous surveillance, l'économie tourne au ralenti et les équipements urbains font défaut.

Les Jeunesses Chrétiennes Maritimes (JMC), créées dans les années 1930 par le Père Lebret, constatent que le manque d'association et de structure réduit les jeunes des ports à une inactivité qui inquiète l'occupant. En 1941, une réunion de cinquante délégués décide d'organiser des Centres de formation manuelle et pratique, dispensant une culture générale axée sur la mer. Ils reprennent les idées des Centres d'études coopératifs maritimes fondés au Havre en 1937, qui soulignaient la nécessité de former les marins face à l'évolution des techniques et des conditions de travail à bord. Obtenant une subvention de l'État de 80000 francs, les J.M.C. créent deux premiers établissements quelques semaines après leur réunion.

Les Écoles d'apprentissage maritime se multiplient en zone "occupée ", d'autres en zone libre sous le contrôle du Bureau de l'apprentissage maritime à Paris. Les J.M.C. cherchent des personnalités (officiers, armateurs, marins, syndicalistes) pour prendre en charge l'institution.

Sous le gouvernement de Vichy, le 28 Juillet 1941, le Journal officiel annonce la constitution de l'Association pour la gérance des écoles d'apprentissage maritime (AGEAM) selon la loi de 1901 sur les associations, dont le secrétaire général est à Paris, et le secrétaire adjoint à Marseille. René Tannay, secrétaire général, et compagnon de route du Père Lebret dans les années 1930, décrit la naissance de l'Association dans son premier bulletin Liaison : "A l'origine, une équipe de jeunes marins récemment démobilisés, qui s'inquiètent des conditions de vie difficiles imposées aux jeunes du milieu maritime dans la période qui suit l'armistice ». Il poursuit : « Le ministère de la mer s'occupant à ce moment de venir en aide aux jeunes de divers milieux sociaux, nos amis orientent leurs efforts dans ce sens. Mais l'adaptation ne se fait pas assez rapidement et l'essai de solutions ne peut se mener qu'avec la jeunesse ».

49 Les statuts de l'association stipulent qu'elle a pour objet de promouvoir et de gérer toutes les réalisations tendant à favoriser l'apprentissage maritime, éduquer, rééduquer, orienter et distraire les jeunes marins, en attendant que cette charge puisse être assurée par les corporations maritimes.

En février 1942, dans le numéro 3 de Liaison, René Tannay fait le point des vingt-quatre écoles : «Cette période de mise en route effectuée dans un moment difficile nous a fait découvrir de nombreux obstacles ». Le manque de matériel et de fournitures, de locaux, la rareté de l'outillage ne facilitent pas la création d'ateliers. Les EAM risquent de devenir de simples écoles privilégiant la théorie plutôt que la pratique et ne répondant pas aux besoins des élèves qui en avaient « déjà plein le dos de la théorie scolaire». 
L'AGEAM, en accord avec l'Amirauté, cherche les moyens de rendre l'apprentissage maritime obligatoire. Une circulaire ministérielle du 31 mars 1941 autorisait tout organisme professionnel (Chambres de commerce et d'industrie, communauté de pêcheurs, municipalité) à créer et gérer des écoles : vingt dépendaient de l'AGEAM, sept du Groupement des capitaines au long cours de la transat, tandis que La Rochelle et Dieppe organisaient leurs écoles locales. Une autre circulaire ministérielle du 29 juillet 1946 confie l'ensemble des écoles à l'AGEAM en modifiant son conseil d'administration. En 1941-42, on comptait 409 élèves; en 42-43, 713 et en 43-44, 711. Le 1er janvier 1943, l'AGEAM avait vingt-sept écoles, deux en Méditerranée et sept en Algérie, celles de France étant implantées au hasard des locaux que l'occupant n'avait pas réquisitionnés.

51 L'enseignement hebdomadaire de trente-cinq heures (vingt heures de travaux manuels et d'exercices de mer, dix de cours théoriques et d'instruction générale, cinq d'éducation physique) occupait les élèves répartis en "canots» de douze élèves. L'organisation des écoles était calquée sur celle des navires.

52 Accusés d'entretenir «le règne du nœud et de l'épissure ", les EAM se défendaient en avançant l'argument qu'elles ne prétendaient pas faire des spécialistes en douze semaines, mais qu'il s'agissait pour elles de répondre à une insuffisance de connaissances requises pour le service de bord, de dégrossir le travail et de permettre aux jeunes de se rendre utiles dès leur embarquement. Les enseignants, professionnels en activité pour les matières pratiques, suivaient des stages de formation et des conférences. Les directeurs, issus de la profession, y retournant, revenant à la direction des écoles, pouvaient aussi enseigner quelques heures par semaine dans les matières proches de leur spécialité. Cette alternance entre activité professionnelle et enseignement apparaissait comme un atout important face aux intentions de l'Enseignement technique d'annexer l'apprentissage maritime. Notons que durant cette période, les EAM ont été considérées comme des "leurres pour l'occupant", permettant aux jeunes, avec l'aide de la Direction des gens de mer qui distribuait des certificats d'emploi officiel, d'échapper au service du travail obligatoire (STO).

Tout au long de son histoire, l'AGEAM a connu une succession d'événements plus ou moins heureux et d'attaques toujours justifiées par et pour ceux qui les engageaient. Personnalités extérieures et personnels de l'association lui ont reproché son déficit financier chronique qu'ils imputaient à une mauvaise gestion, tandis que l'amélioration des locaux et des moyens pédagogiques était négligée. Par ailleurs, le niveau de formation des écoles a souvent été remis en cause. Une série de stages privés (maisons familiales, organismes divers ainsi que la création de sections conchylicoles dans les établissements publics) ont sans cesse fait planer une menace sur l'existence même des EAM.

54 Enfin la profession elle-même, qui, lorsqu'elle était en crise, réclamait des formations afin d'assurer sa survie, les rejetait lorsque l'exigence de diplôme pour l'exercice du métier ou l'obtention des concessions et de dotation d'aides pour l'installation d'entreprises conchylicoles risquait d'amener sur le marché du travail un certain nombre de jeunes extérieurs au milieu qui échapperaient à l'autorité des anciens.

Pour répondre à cette concurrence sur le marché de la formation, l'AGEAM crée en 1984 le certificat d'aptitude professionnelle (CAP) de marin pêcheur. L'arrêté du 8 juin 1975 fait obligation à tout marin quel que soit son âge, de justifier d'un titre de formation professionnelle pour embarquer sur un navire de plus de 25 tonneaux. En 1991, un arrêté fait obligation d'un diplôme pour tout embarquement à la pêche quelle 
que soit la jauge du navire. Pourtant, en 1988 encore, on peut lire dans le numéro 4 de France Pêche, que formateurs et professionnels jugent le CAP trop long, car trop théorique pour des élèves issus pour beaucoup de Sections d'enseignement spécialisé ou de CPPN (Classes préprofessionnelles de niveau). Ces propos sont jugés par les responsables de l'Association pour la gérance des écoles maritimes et aquacoles (AGEMA) et les instances ministérielles comme le résultat d'une mentalité archaïque qu'il convient de changer.

En 1985, en application des dispositions de la loi de décentralisation, les EAM deviennent des Écoles maritimes et aquacoles (EMA), l'AGEAM devient l'AGEMA. La récente réforme Loquet (1989) supprime l'ancien certificat d'apprentissage maritime (CAM) qui donnait accès, après neuf mois d'école, aux fonctions de novice ou de matelot à bord. Le CAP rénové développe la formation en stage embarqué et l'enseignement des techniques de gestion et d'informatique. L'année scolaire 1991-92 est marquée par la mise en route du brevet d'études professionnelles (BEP) pêche dont les premiers résultats ne seront connus qu'à la fin de l'année scolaire 1992-93. Le diplôme du Bac professionnel est alors à l'étude. Le but de la réforme est de développer un véritable cursus CAP-BEP-BAC permettant d'accéder à une formation plus ciblée. Cette transformation des contenus de formation entend répondre à la situation de la pêche dans un contexte de politique commune des pêches, caractérisée par une raréfaction de certaines ressources entraînant les États membres à faire diminuer l'effort de pêche et à augmenter la qualité des produits et la rationalité de la gestion des stocks et des entreprises. Par ailleurs, au niveau des statuts, les EMA deviennent sous l'effet de la loi de décentralisation, des EPLE (Établissements publics locaux d'enseignement), statut leur assurant l'autonomie administrative et financière, en les faisant passer sous la houlette de la Région. La pêche, durant cette récente période, est caractérisée par une entrée dans la technologie de pointe mais aussi par la permanence de pratiques jugées archaïques, caractéristiques opposées qui traversent et animent les débats et les actions concernant la formation.

Conclusion

57 Jusqu'à la fin du XIX ${ }^{e}$ siècle, aucun diplôme n'était requis pour devenir pêcheur. On apprenait enfant en accompagnant les parents sur la grève dans le ramassage des coquillages et poissons, puis en embarquant avec le père au fur et à mesure que la pêche s'est éloignée du rivage. Ce n'est qu'en 1897 que le Certificat d'Étude est exigé pour les enfants de dix à treize ans qui souhaitent s'inscrire sur un rôle d'équipage. Au début $\mathrm{du} \mathrm{xx}^{\mathrm{e}}$ siècle, les diplômes sont réclamés aux candidats au brevet de patron de pêche.

En 1975, l'exigence de diplôme ne concerne que les marins désirant être portés sur le rôle d'un navire de plus de 25 tonneaux. L'arrêté du 24 juillet 1991 abroge celui du 8 juin 1975 et exige un titre de formation professionnelle quelle que soit la jauge du navire (mais avec des dérogations selon l'âge et l'expérience). Ces mesures visent à réduire la flotte des petites unités de pêche qui s'accroît en sens inverse de la flotte industrielle, alors que l'Europe bleue s'efforce de réduire l'effort de pêche.

D'entreprise humanitaire visant à améliorer les conditions de vie des marins, la formation professionnelle est devenue l'outil de la profession organisée cherchant à contrôler son marché de l'emploi (Vourc'h 1980). Ce sont les cadres de la profession qui étaient et sont membres des conseils d'administration des écoles de pêche, des comités locaux des pêches... 
60 Aujourd'hui, la profession se transforme en filière où l'activité de pêche englobe la capture, la transformation et la commercialisation. Ses écoles, faites par et pour les pêcheurs, doivent adapter leurs contenus pédagogiques et diversifier leurs enseignements. Si on ne parle plus depuis longtemps d'école de pêche, il n'est plus question désormais que de lycées maritimes et aquacoles.

\section{BIBLIOGRAPHIE}

Biget, Denis

1992. Entre l'école et la mer. Motivations et représentations des jeunes en formation dans les

Écoles maritimes et aquacoles en Bretagne. Mémoire de maîtrise, sous la direction de P.-J. Simon. Université Rennes II, Département de sociologie.

1997. Histoire des écoles de pêche. Contribution à une ethnosociologie des populations littorales. Spézet : Coop Breizh.

Cabantous, Alain

1991. Dix mille marins face à l'océan. Les populations maritimes de Dunkerque au Havre aux XVII et XVIII ${ }^{\mathrm{e}}$ siècles (vers 1660-1794). Paris : Publisud (« Études sociales »).

Delbos, Geneviève \& Paul Jorion

1984. La Transmission des savoirs. Paris : Éditions de la Maison des sciences de l'Homme.

Durkheim, Emile

1922. Éducation et sociologie. Paris : Presses Universitaires de France.

Duviard, Dominique

1992. Groix, l'île des thoniers ; chronique maritime d'une île bretonne. Atelier Alpha Bleue.

Guillard, Victor

1896. «École de pêche de Groix », Bulletin des Pêches Maritimes n4.

Mollat, Michel (ed.)

1988. Histoire des pêches maritimes en France. Toulouse : Privat.

Prost, Antoine

1968. Histoire de l'enseignement en France (1800-1967). Paris : Armand Colin.

Raymond, Marie-Geneviève

1968. «Idéologie du logement et opposition ville-campagne », Revue Française de Sociologie IX :

167-179.

Roignant, Jacques

1992. Le Quartier maritime de Lannion. Contribution à l'histoire des pêches en baie de Lannion.

Rougier-Pintiaux, Pascale

1988. « Les instituteurs du XIX ${ }^{\mathrm{e}}$ siècle et le travail manuel », Revue française de sociologie XXIX-2 :

275-292. 
Vauclare, Claude

1988. « Naissance d'une industrie », pp. 243-296, in M. Mollat (ed.), Histoire de pêches maritimes en

France. Spezet : Nature et Bretagne.

Vourc'h, François

1980. Analyse des rapports sociaux à la pêche artisanale. Histoire et évolution. Rapport CORDES

VI.

\section{NOTES}

1. La philosophie de la loi du 28 mars 1882 qui impose l'enseignement des travaux manuels dans les écoles primaires est à l'opposé de la philosophie de l'enseignement pour le métier qui animera les écoles de pêche. Pour une étude comparative de ces deux courants pédagogiques et idéologiques, lire Rougier-Pintiaux (1988) et Prost (1968).

2. D'autres institutions s'emploient à cette mission, telle que l'École maritime de Port Hallan à Belle-île qui dépend de l'Assistance Publique et reçoit des enfants pour leur donner un enseignement nautique au commerce et à la pêche, avec engagement volontaire au service des équipages de la flotte.

3. Groix compte à la fin du XIX ${ }^{\mathrm{e}}$ siècle 277 voiliers, ce qui en fait le premier port thonier. 4. Les archives exploitées ne contiennent que des allusions sur ces questions qu'il sera éclairant d'approfondir par une analyse du contexte local pour chaque école.

\section{RÉSUMÉS}

En 1895, les fondateurs des écoles de pêche cherchent à sortir les marins pêcheurs de l'archaïsme et de l'empirisme pour les conduire à plus de rationalité dans leur pratique. Jusque-là, la pêche s'est développée entre archaïsme et modernisme. Autrefois, le pêcheur devait apprendre son métier par apprentissage, en reproduisant ce qu'il observait sur le bateau. Le certificat était juste une formalité. Désormais, on demande au pêcheur d'apprendre son métier de façon rationnelle et scolaire afin de répondre aux exigences de l'économie de marché. C'est aussi une façon d'encadrer la profession et de permettre à l'État de contrôler cette part de l'activité économique nationale et cette partie des populations littorales.

From fishing schools to maritime and « aquacultural » colleges.

In 1895 , founding members of teaching establishments in the fishing sector sought to extract fishermen from their culture of archaism and empiricism, and to encourage a greater degree of rationality in their professional practice. Until this time, fishing had evolved in the mid-ground between archaism and modernism. In the past, the fishermen had to learn their trade through observation and then put into practice what they have seen. The certificate was just a formality. From then on, fishermen were expected to learn their trade in a rational and academic way in order to be able to meet the demands of the market economy. Formal training also provided a mean of monitoring the profession, affording the state a degree of control over this part of the national economy and this particular coastal population. 
De las escuelas de pesca a los liceos marítimos y las « acuácolas ».

En 1895, los fundadores de las escuelas de pesca se propusieron arrancar a los marineros del arcaísmo y el empirismo en que estaban sumidos con el objetivo de guiarlos hacia una práctica más racional. Hasta entonces la pesca se había venido desarrollando a medio camino entre arcaísmo y modernidad. Antaño, el pescador debía aprender su oficio a través del aprendizaje, reproduciendo aquello que observaba mientras estaba embarcado. El certificado constituía un simple formalismo. A partir de ahora, se exigirá del pescador que aprenda su oficio de manera racional y escolar, a fin de poder responder a las exigencias de la economía de mercado. Esta era una manera de encuadrar a la profesión y de permitir al Estado controlar esta parte de la actividad económica nacional y esta fracción de las poblaciones litorales.

INDEX

Mots-clés : histoire, apprentissage, écoles de pêche, institution, métier

Keywords : history, apprenticeship, fishing school, trade

\section{AUTEUR}

DENIS BIGET

Département d'anthropologie, d'ethnologie et de science des religions. Université Paris VII. 